\title{
BMJ Open Partial versus complete prostatectomy specimen sampling: prospective non- inferiority study for pT3a tumours and surgical margin involvement
}

\author{
Eelco R P Collette, ${ }^{\oplus 1}$ Michael A den Bakker, ${ }^{\odot}$ Sjoerd O Klaver, ${ }^{3}$ André N Vis, \\ Mike Kliffen ${ }^{2}$
}

To cite: Collette ERP, den Bakker MA, Klaver S0, et al. Partial versus complete prostatectomy specimen sampling: prospective noninferiority study for $\mathrm{pT} 3 \mathrm{a}$ tumours and surgical margin involvement. BMJ Open 2019;9:e024524. doi:10.1136/ bmjopen-2018-024524

- Prepublication history for this paper is available online. To view these files, please visit the journal online (http://dx.doi org/10.1136/bmjopen-2018024524).

Received 30 May 2018 Revised 13 January 2019 Accepted 7 March 2019

Check for updates

(c) Author(s) (or their employer(s)) 2019. Re-use permitted under CC BY-NC. No commercial re-use. See rights and permissions. Published by BMJ.

${ }^{1}$ Urology, VU medisch centrum School of Medical Sciences, Amsterdam, The Netherlands ${ }^{2}$ Pathology, Maasstad

Ziekenhuis, Rotterdam, The Netherlands

${ }^{3}$ Urology, Maasstad Ziekenhuis, Rotterdam, The Netherlands

Correspondence to

Dr Eelco R P Collette;

eelco@collette.nl

\section{ABSTRACT}

Aims The importance of additional information gained by complete versus partial sampling or prostatectomy specimens is uncertain. There is sparse data on the value of complete versus partial sampling and numbers of inclusions in studies are small and retrospective. We present the results of a prospective non-inferiority study to examine if partial sampling is inferior to complete sampling in terms of pathology outcomes and clinical relevance.

Methods 564 robot-assisted prostatectomy (RARP) specimens with prospective registration and analysis were collected over a 2-year period. All patients underwent RARP between January 2014 and February 2016 in our hospital after a diagnosis of clinically localised prostate cancer. For each patient, tumour stage and surgical margin status was recorded after partial and after complete sampling. Upstaging from pT2 to pT3a and upgrading from a negative-to-positive surgical margin was analysed. Results In 12 of 564 patients (2.1\%), complete sampling yielded new information. In eight patients (1.4\%), the surgical margin converted to positive after complete sampling. Upstaging from initial pT2 tumour in partial sampling to $\mathrm{pT} 3 \mathrm{a}$ tumour after complete sampling was documented in five patients $(0.9 \%)$. In the follow-up period (mean 35 months), a biochemical recurrence occurred in one patient.

Conclusions Complete sampling provides new information in only $2.1 \%$ of cases, compared with partial sampling. We conclude that the additional information gained by complete sampling in terms of stage and surgical margin detection is statistically insignificant compared with partial sampling. Furthermore, partial sampling compared with complete sampling does not change postoperative clinical management.

\section{INTRODUCTION}

The most commonly diagnosed solid-organ cancer is prostate cancer ( $\mathrm{PrC}$ ) and it is the second most cause of cancer-related deaths in men. ${ }^{1}$ Radical prostatectomy (RP) is the treatment of choice for patients with clinically localised PrC who have a life expectancy over 10 years. $^{2}$ RP can be performed
Strengths and limitations of this study

- Sample size sufficiently large to power this study

- Robust protocol.

- Additional possible prognostic factors were not assessed such as Gleason score and extend of extraprostatic extension.

- No formal cost analysis was performed.

- In many laboratories, large blocks are currently preferred over standard size blocks, which may mitigate the increased workload reported in this paper.

as an open or laparoscopic procedure. In Western countries, adoption of the robotic platform has increased tremendously since its launch in $2000 .{ }^{3}$ One of the purposes of pathological examination of RP specimens is determination of positive margins and identification of T3a tumours, because these are important predictors of biochemical recurrence. However, it is currently not known how many additional cases with positive margins or a pT3a stage will be found by complete versus partial embedding and whether complete sampling is superior in this respect. The consequence of complete embedding of all prostatic tissue in comparison to partial embedding is that over $50 \%$ more tissue has to be sampled, processed and examined. In particular when standard size histology blocks are used, which is commonplace in the Netherlands, this results in a significant increase in workload and materials, thus causing an increased burden on resources.

In 2014, revised Dutch national guidelines for PrC diagnosis and treatment were published. ${ }^{4}$

The revised guidelines advocate complete rather than partial sampling of RP specimens. Furthermore, it states that partial embedding is acceptable, but only if at least the entire dorsal side is enclosed. It has been suggested 
that complete embedding of RP specimens is superior for determining stage and the status of the surgical margins. However, partial sampling techniques have been described with comparable results to complete embedding. ${ }^{5-8}$ The assumptions for the recommendations in the revised guidelines may be questioned. Not all available literature was included in the guideline preparation because of perceived inadequate quality owing to insufficient study sample size. Only results from larger studies were used in the guideline revision; thus, the opinion was based on older studies, while newer studies with smaller samples which did not demonstrate superiority of complete embedding were discarded. In addition, in the dated studies, with larger numbers of samples larger tumours were investigated, potentially introducing a confounding factor. We hypothesise that complete sampling provides little additional prognostic information, takes more time, costs more money and has little influence on patient outcome and clinical decision-making.

Prior studies have compared different methods for examining prostatectomy specimens. ${ }^{5-16}$ However, the majority of these studies lack follow-up data, which is of paramount importance to determine the optimal processing protocol. Furthermore, many different embedding protocols are described in published studies which hampers direct comparison.

In the work presented here, direct comparison of two embedding protocols was performed in a large and prospective series of RP specimens. Here, we compare complete embedding (ie, submission of all tissue for histology) as advocated in guidelines, to partial embedding, where a limited amount of tissue is submitted for histology. The objective of this study was to evaluate complete versus partial RP specimen sampling for tumour stage, surgical margins and to correlate these parameters with clinical outcome. The study was designed as a non-inferiority set-up aimed at rejecting the hypothesis that partial embedding is inferior to complete embedding.

\section{MATERIAL AND METHODS}

All patients who underwent a robot-assisted radical prostatectomy (RARP) in our hospital between January 2014 and February 2016 were included in this study. There were no exclusion criteria; all patients were included. Patients with a biopsy diagnosis of $\mathrm{PrC}$ were worked up according to standard hospital procedures. Patients underwent RARP after a diagnosis of clinically localised PrC. All RARP operations were performed by experienced urologists using the da Vinci robotic surgical system (Intuitive Surgical, California, USA). The robotic programme started in 2009 in our hospital. The study was approved by the local ethical committee. Patients and public were not involved in the development of the research question or in the design of this study.

All tissue samples were treated as routine pathology specimens. The tumour grade, stage and surgical margin status were evaluated according to standard

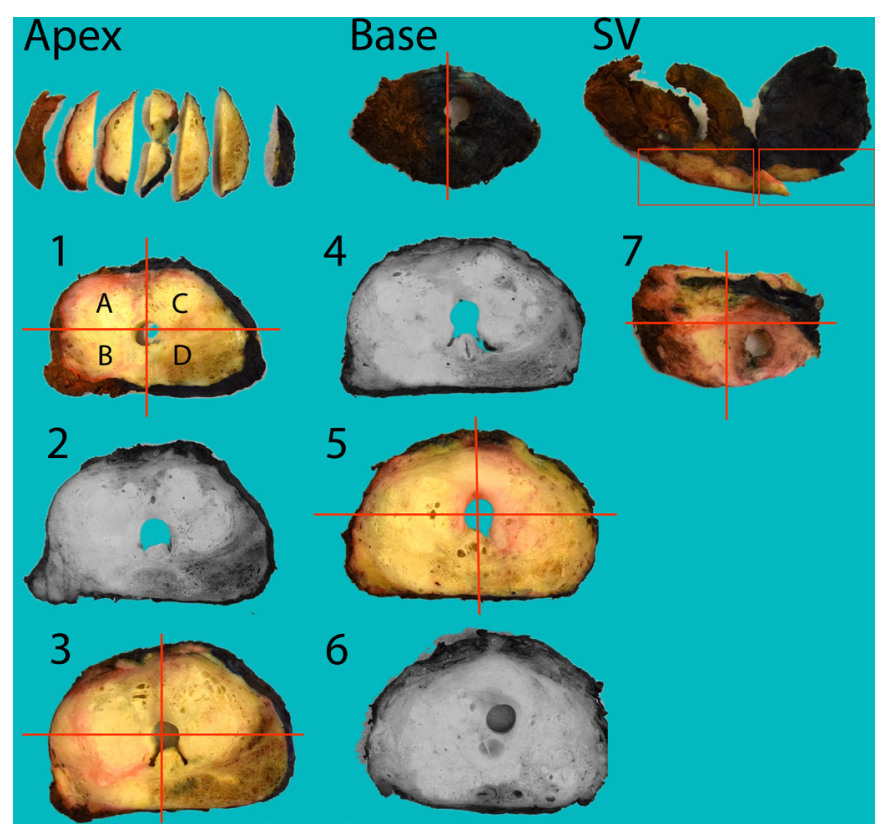

Figure 1 Semidiagrammatic example of robot-assisted radical prostatectomy processing. After fixation and inking, the apex is removed and cross sectioned. The base is removed and embedded as shave sections. The seminal vesicles (SVs) are removed, and the base of each vesicle is embedded separately. The gland is sectioned from apex to base (in this example, in seven 3-4 mm slices) numbered from apex to base. Each slice is cut in four quadrants (A-D). Quadrants of odd numbered sections $(1,3,5,7)$ are embedded as partial sampling. For complete sampling, even numbered slices $(2,4,6)$ are then embedded. Results from partial and complete embedding were recorded separately; the final pathology report was based on complete embedding.

guidelines. ${ }^{47-19}$ For each patient, the pathology result was registered after partial sampling and after complete sampling in one central prospective database. The routine pathology report was based on the completely embedded specimen. Upstaging from pT2 to pT3a and upgrading from a negative to a positive surgical margin was analysed. The end terms are additional cases of pT3a tumour or positive surgical margin after complete sampling.

The pathological processing protocol entailed standard partial embedding with subsequent further embedding and processing of all additional prostate tissue (figure 1). A standardised protocol was used consistently throughout the study. We did not select specific slices due to suspicious lesion for cancer at gross examination. Processing of the specimens was performed by pathologists or pathology assistants after training and supervision. All specimens were received in neutral buffered formalin. On receipt, the specimens were inked, weighed and measured and fixed for 24 hours. Embedding and histological processing were performed with standard size histological cassettes.

After fixation, for the standard procedure (partial embedding), the apex shave section was sliced perpendicular to the shave surface and embedded; shave sections (left and right sides) of the base were embedded en 
face on the cut surface. The remainder of the specimen was sliced from apex to base in an equatorial plane in 3-4 mm thick slices, numbered from apex to base. Odd numbered $(1,3,5$, etc) slices were embedded in quadrants (left anterior, left posterior, right anterior and right posterior). A single section through the base of each seminal vesicle was embedded. Sections embedded up to this point comprise our standard protocol. For complete embedding, the remaining even numbered slices were embedded in a similar way. For very large specimens which could not be embedded in quadrants, the slice was divided and embedded in six sections (left anterior, left posterior, central anterior, central posterior, right anterior and right posterior). Any additional tissue embedded separately was considered part of the standard set and handled as such. Standard hematoxylin and eosin (H\&E)stained slides were prepared and evaluated.

Extraprostatic extension (EPE) was determined and measured as described by Magi-Galluzzi et al. ${ }^{17}$ Briefly, the radial expansion of tumour cells perpendicular to the perimeter of the prostate with tumour cells infiltrating in periprostatic fat was measured and recorded. A surgical margin was considered involved only when tumour cells were in direct contact with the marking ink, as recommended by Tan et al. ${ }^{19}$ The cumulative extent of margin involvement was recorded in $\mathrm{mm}$. A diagnostic synoptic pathological report was filed for the complete set of slides. For the study protocol, separate parameters were recorded for the standard set of (paraffin) blocks and the additional blocks. In addition to standard items, additional recorded parameters for both sets comprised number of slices, number of blocks, tumour volume percentage, radial EPE and resection margin status (measured in $\mathrm{mm}$ if involved). A separate note was made if the additional sections of complete embedding provided new finding over the standard blocks of partial embedding. Data were prospectively recorded in a database.

Statistical sample size calculation was performed using PASS V.12.0 (NCSS Statistical Software, Kaysville, Utah, USA). The study was designed for a maximum clinical difference or margin of equivalence of $5 \%$, which is our threshold for the non-inferiority range. We performed a power analysis of non-inferiority tests of correlated proportions for numerical results of a non-inferiority (one-sided) test of a difference. A sample size of 349 subjects achieves $90 \%$ power at a $1 \%$ significance level. A calculated $90 \%$ CI by Wald $\mathrm{Z}$ methods ranges from -0.029 to -0.014 , with $\mathrm{p}<0.05$. In other words, if the difference between partial and complete sampling falls in the CI of $1.4 \%$ and $2.9 \%$, it is within the statistical margin of equivalence and thus partial embedding is non-inferior with a $\mathrm{p}$ value of $<0.050 .^{20}$

\section{RESULTS}

The patient characteristics are summarised in table 1.

Over a 26-month timespan, 564 consecutive RARP specimens were collected and were processed according

\begin{tabular}{|c|c|}
\hline Variable & \\
\hline Age (years) & 67 (median) (range 41-80) \\
\hline Referred patients & $452 / 564(80 \%)$ \\
\hline Local patients & $112 / 564(20 \%)$ \\
\hline Prostate weight (grams) & $64(20-192)$ \\
\hline Initial PSA ( $\mu \mathrm{g} / \mathrm{L})$ & $\begin{array}{l}8.9 \text { (median) (range 1.5- } \\
82.0 \text { ) }\end{array}$ \\
\hline cT1c (\%) & $327 / 564(58 \%)$ \\
\hline cT2abc (\%) & $215 / 564(38 \%)$ \\
\hline cT3 (\%) & 22/564 (4\%) \\
\hline $\begin{array}{l}\text { Biopsy Gleason } 3+3=6 / G^{*}-1 \\
(\%)\end{array}$ & $276 / 564(49 \%)$ \\
\hline $\begin{array}{l}\text { Biopsy Gleason 3+4=7/GG-2 } \\
\text { (\%) }\end{array}$ & $143 / 564(25 \%)$ \\
\hline $\begin{array}{l}\text { Biopsy Gleason 4+3=7, 8, 9, } \\
\text { 10/GG-3, 4, } 5 \text { (\%) }\end{array}$ & $145 / 564(26 \%)$ \\
\hline рT2 (\%) & $400 / 564(71 \%)$ \\
\hline pT3ab (\%) & $164 / 564(29 \%)$ \\
\hline $\begin{array}{l}\text { Pathological Gleason 3+3=6/ } \\
\text { GG-1(\%) }\end{array}$ & $216 / 564(38 \%)$ \\
\hline $\begin{array}{l}\text { Pathological Gleason } 3+4=7 / \\
\text { GG-2 }(\%)\end{array}$ & $181 / 564(32 \%)$ \\
\hline
\end{tabular}

Pathological Gleason 4+3=7, 8, 167/564 (30\%)

9, 10/GG-3, 4, 5 (\%)

Total patients 564

${ }^{*} \mathrm{GG}$, grade group (International Society of Urological Pathology);

PSA, prostate specific antigen.

to the study protocol. Included in the study was the total RARP hospital volume. Standard processing generated an average of 6.8 slices (range 4-11; median 7) per prostate, including apex and base, resulting in an average of 27.2 blocks (range 14-72; median 26). Additional processing to attain complete embedding generated an average of 4.4 slices (range 4-9; median 4) per prostate and an average of 18.7 blocks (range 8-72; median 16). In total, the 564 cases generated 15335 standard protocol blocks and 10547 extra blocks (table 2). The additional processing resulted in an increased workload of $65 \%$ in slices and $69 \%$ in blocks. Tumour tissue was identified in all partially embedded specimens.

\begin{tabular}{|c|c|c|c|}
\hline$n=564$ & $\begin{array}{l}\text { Partial } \\
\text { sampling }\end{array}$ & $\begin{array}{l}\text { Complete } \\
\text { sampling }\end{array}$ & Difference \\
\hline $\begin{array}{l}\text { Slices average (range; } \\
\text { median) }\end{array}$ & $\begin{array}{l}6.8(4-11 ; \\
7)\end{array}$ & $\begin{array}{l}+4.4(4-9 \\
4)\end{array}$ & $+65 \%$ \\
\hline $\begin{array}{l}\text { Blocks average (range; } \\
\text { median) }\end{array}$ & $\begin{array}{l}27.2(14- \\
72 ; 26)\end{array}$ & $\begin{array}{l}+18.7 \\
(8-72 ; 16)\end{array}$ & $+69 \%$ \\
\hline Total blocks & 15335 & +10547 & $+69 \%$ \\
\hline
\end{tabular}


Table 3 Tumour stage and surgical margin involvement in partial versus complete prostate specimen sampling

\begin{tabular}{|c|c|c|c|}
\hline$n=564$ & $\begin{array}{l}\text { Partial } \\
\text { sampling }\end{array}$ & $\begin{array}{l}\text { Complete } \\
\text { sampling }\end{array}$ & Difference \\
\hline pT3a \% (N) & $\begin{array}{l}23.6 \% \\
(133 / 564)\end{array}$ & $\begin{array}{l}24.5 \% \\
(138 / 564)\end{array}$ & $0.9 \%(5)$ \\
\hline $\begin{array}{l}\text { Positive surgical } \\
\text { margin \% (N) }\end{array}$ & $\begin{array}{l}33.2 \% \\
(187 / 564)\end{array}$ & $\begin{array}{l}34.6 \% \\
(195 / 564)\end{array}$ & $1.4 \%(8)$ \\
\hline $\begin{array}{l}\text { pT3a and/or positive } \\
\text { surgical margin \% (N) }\end{array}$ & $\begin{array}{l}39.4 \% \\
(222 / 564)\end{array}$ & $\begin{array}{l}41.5 \% \\
(234 / 564)\end{array}$ & $2.1 \%(12)$ \\
\hline
\end{tabular}

In 12 cases $(2.1 \%)$, the additional blocks provided new information (tables 3-5).

In eight cases $(8 / 564=1.4 \%)$, the extra blocks revealed an involved resection margin. Partial embedding only missed insignificant surgical margins, ranging from 1 to 3 $\mathrm{mm}$. In five cases $(5 / 564=0.9 \%)$, the extra blocks showed EPE which was not present in the standard blocks. Partial embedding only missed focal EPE, ranging from 0.7 to $1 \mathrm{~mm}$. Upstaging from an initial pT2 tumour in partial sampling to a pT3a tumour or revealing an involved surgical margin after complete sampling was documented in 12 patients $(12 / 564=2.1 \%)$. These 12 patients have a follow-up period ranging from 26 to 42 months; in one patient, a biochemical recurrence occurred after 19 months of follow-up and he received additional radiation treatment for local control. Test parameters of partial sampling (with complete sampling as 'gold standard') show a sensitivity of $94.9 \%$, a specificity of $100 \%$ with a positive predictive value of $100 \%$ and a negative predictive value of $96.5 \%$.

\section{DISCUSSION}

Several histological criteria of cancer in prostatectomy specimens have been shown to have prognostic implications. These features include differentiation (as assessed by Gleason score and Gleason grade group), margin status, invasion beyond the prostate gland (EPE, stage) and invasion of seminal vesicles. These features are commonly only focally present in the resected gland. This raises the question of how much effort should be made to identify very small areas with adverse prognostic factors, which may be of no importance. This question underpins the debate of how much tissue should be examined for adequate and relevant grading and staging and which protocol should be adopted. ${ }^{20} 21$ The obvious benefit of partial embedding versus complete embedding of RP specimens is a reduction in costs afforded by fewer tissue samples requiring pathological processing and consequently fewer histological slides which require reporting by the pathologist. The question arises whether and at which point limiting the amount of tissue which is investigated through partial embedding negatively impacts on observed pathological parameters and may ultimately impact on patient management and disease outcome. While the question of 'how much tissue should be examined' is valid for any tumour, it is highly relevant for $\mathrm{PrC}$, as this is often not grossly visible and therefore the macroscopic appearance is unsuitable to guide tissue sampling. It is in this context relevant to realise that even in complete sampling of a prostate only $0.17 \%$ of the prostatic tissue is microscopically studied and partial sampling reduces this by $40 \%$ to approximately $0.1 \% .^{13}$ The consequence of these established facts is that slicing of the gland for histological processing is not overtly critical and does not need to be rigidly controlled as has been suggested. ${ }^{5}$ Despite these sobering realisations, it remains uncertain what the optimal processing strategy is for RP specimens. The benefits and drawbacks of complete and partial embedding have been addressed in a number of studies with conflicting results (table 6 ).$^{5-16}$

Comparison of the studies is hampered by the fact that (1) different partial embedding protocols are used; (2) comparison groups were often not matched for tumour parameters and (3) no- or different outcome measures were applied. Nevertheless, the majority of the studies conclude that partial embedding is acceptable with marginal lower levels of surgical margin detection and accuracy of staging. Data on outcome are variable; while positive margin status is expected to increase with complete sampling, this does not necessarily result in a worse outcome. ${ }^{5}$

The 2014 Dutch prostate cancer guidelines, and the European Association of Urology guidelines of 2016 recommend complete embedding, while partial embedding may be considered, particularly for larger prostates. ${ }^{24}$

Table 4 Cross table of upgrade to pT3a and/or involved SM

\begin{tabular}{|c|c|c|}
\hline$n=564$ & $\begin{array}{l}\text { Complete sampling: <pT3a and } \\
\text { non-involved SM* }\end{array}$ & $\begin{array}{l}\text { Complete sampling: } \geq \mathrm{pT} 3 \mathrm{a} \text { and/ } \\
\text { or involved SM }\end{array}$ \\
\hline Partial sampling: $<\mathrm{p} T 3 \mathrm{a}$ and non-involved SM & 330 & 12 \\
\hline Partial sampling: $\geq \mathrm{pT} 3 \mathrm{a}$ and/or involved SM & 0 (not available) & 222 \\
\hline Sensitivity & $94.9 \%$ & \\
\hline Specificity & $100.0 \%$ & \\
\hline Positive predictive value & $100.0 \%$ & \\
\hline Negative predictive value & $96.5 \%$ & \\
\hline
\end{tabular}

*SM, surgical margin. 


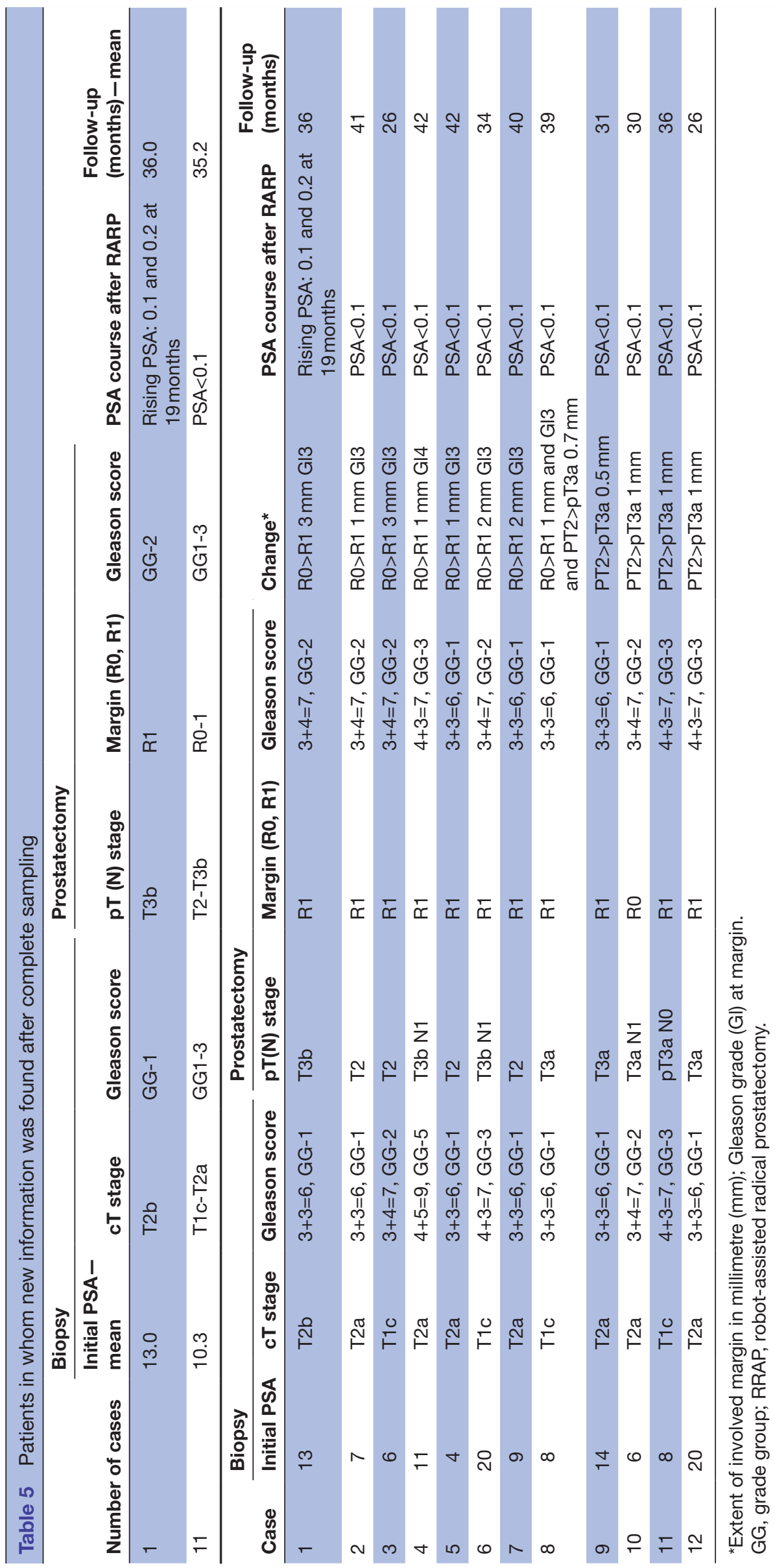

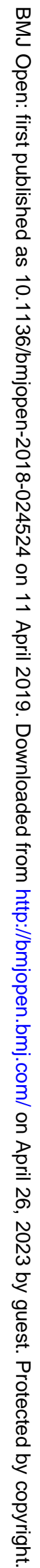


Table 6 Overview of literature

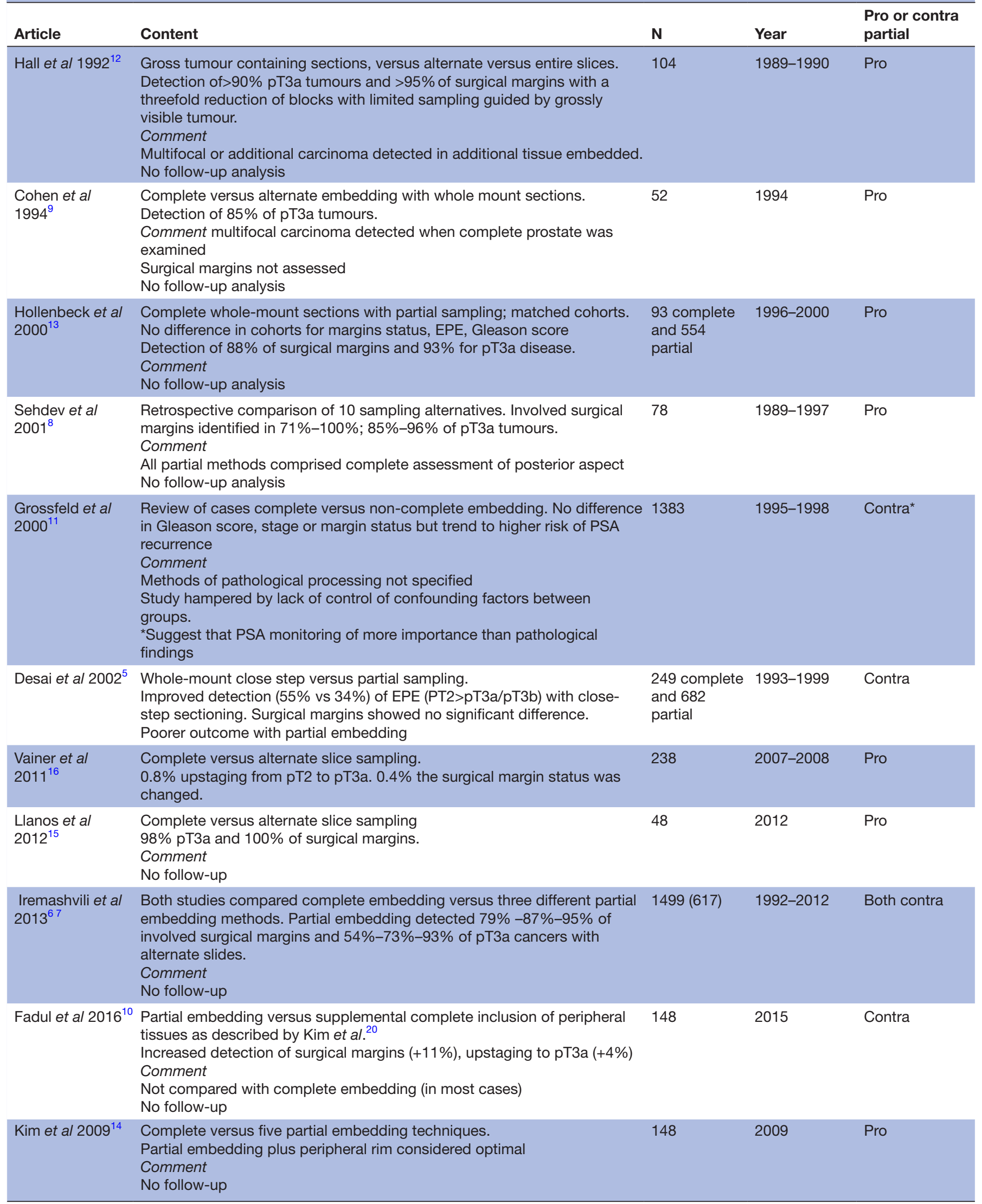

EPE, extraprostatic extension. 
The rationale for these recommendations is still partially based on older retrospective studies and the fact that $\operatorname{PrC}$ is often multifocal and may be grossly indistinct. To fully address the question whether partial embedding of RP specimens yields inferior results compared with complete embedding, we prospectively analysed a large consecutive cohort of 564 RP cases, collected over a 26-month period. To our knowledge, this is the first prospective investigation which critically assesses the value of comprehensive prostatectomy specimen sampling study in RARP. The cohort of patients reflects the Dutch situation with well-balanced classification of clinical malignant tumour (cTNM) stages and pathological outcomes with 34.6\% positive surgical margins and $24.5 \%$ pT3a tumours. The processing protocol, which is consistent with that of other laboratories in the Netherlands, consisted of complete transverse slicing of the gland and embedding all prostatic tissue. Alternate slices were assessed as partial embedding, and inclusion of the remaining slices resulted in complete embedding.

Our results show that complete sampling compared with partial sampling yields new pathological information in $2.1 \%$ of cases. Vainer et al and Llanos et al used similar partial sample techniques in their respective studies with comparable results showing even less than $2 \%$ additional information with complete sampling. ${ }^{15} 16$ However, Iremashvilli et al conclude that even with more tissue embedded in their partial sampling protocol a positive resection margin was missed in $5 \%$ of cases and in $7 \%$ EPE was missed. ${ }^{67}$ In a partial sampling method described by these authors that is similar to ours, using alternate section embedding, $13 \%$ of the positive resection margins and $27 \%$ of EPE were missed. ${ }^{7}$ These figures differ greatly from our results and those of Llanos and Vainer and cannot be fully explained by us, although minor methodological and interpretational differences, such as defining an involved margin (tumour cells close to the margin, versus marking ink actually on tumour cells) may account for part of these discrepancies. A small proportion of our patient population presented with cT3a disease $(4 \%)$, and it is likely that identification of pT3a disease in complete sampling will be even less common if the investigated population already has a high proportion of cT3 patients. In this circumstance, the major advantage of complete embedding is limited to enhanced identification of involved surgical margins.

The study presented here was primarily aimed at determining the potential drawbacks of partial embedding of $\mathrm{RP}$ specimens in comparison to complete embedding in terms of stage determination and surgical margin involvement. Additional parameters which we did not systematically investigate, as these did not directly impact on patient management, could potentially provide additional information. For instance, factors that were not assessed included Gleason score changes, Gleason score at margin, multifocality and tumour volume that have been studied by others, but cannot be compared with this study. Similarly, recording additional features as mentioned above could have been useful for radiological-pathological correlation, in particular if partial resection techniques are introduced in prostate surgery. However, this was not the objective of this study.

In our hospital, RARP specimens generate the greatest number of blocks per submitted specimen type. Prior to this study, partial prostate specimen embedding annually resulted in approximately $12 \%$ of all tissue blocks. Complete embedding substantially increased the number of blocks, bringing the proportion of paraffin blocks produced solely by RP specimens up to $19 \%$ of the total histology workload (of approximately 60000 blocks annually). Bearing in mind the number of RP specimens on an annual basis only forms less than $1 \%$ of all histology submissions, coupled with the limited additional benefit afforded by complete embedding as shown in this study, it is doubtful that complete embedding is justified as a recommended procedure. In our study, standard size histology cassette were used. It is likely that using large ('mega') block cassettes would be more efficient both in terms of processing and reporting. However, even the use of large blocks would not change the findings of our study with regard to staging and surgical margin status.

In The Netherlands prostatectomy patients rarely receive adjuvant radiotherapy administered following the identification of EPE or an involved surgical margin. PSA values are monitored for biochemical recurrence (defined as two consecutive PSA values $\geq 2 \mu \mathrm{g} / \mathrm{L}$ ), in which case early salvage therapy or late salvage therapy is considered. In other words, pathological stage and margin status are not used for direct postoperative treatment decision, but rather for prognosis and patient education. Therefore, our results that reveal additional information on stage and margin status in $2.1 \%$ of patients while requiring $65 \%-69 \%$ additional sampling with associated time and costs have negligible effect on patient management and outcome. In our opinion, this additional workup with limited additional information does not justify the increase in workload, and thus is not cost effective. At our hospital, 275 robotic radical prostatectomies are performed annually, of which approximately $55 \%$ are combined with pelvic lymph node dissection (pLND). For histology processing, the costs of partial sampling of RP specimens without and with pLND are $€ 400$ and $€ 700$, respectively, compared with $€ 600$ and $€ 900$ for complete sampling. Overall, complete sampling increases the cost by $€ 200$ per patient. The sum of hospital costs for sampling of 275 radical prostatectomies increases from $€ 155000$ to $€ 212000$ annually, an increase of $37 \%$. Thus, in the discussion of pathological processing, it is relevant to put this in perspective considering that monitoring PSA levels, at a fraction of the increased cost of complete rather than partial embedding of RARP specimens, may well be of superior importance for recurrence prognostication. However, many additional tumour parameters, which provide important additional prognostic information, are determined solely by the pathological examination of RP specimens. 
We conclude that the additional information gained by complete sampling in terms of stage and surgical margin detection is statistically insignificant compared with partial sampling and has no effect on postoperative clinical management. In this era with a strong focus on valuebased healthcare, we suggest review of the guidelines taking added cost with limited added value into account.

\section{Generalisability}

1. This study shows that partial embedding of RP specimens is not inferior to complete embedding.

2. The majority of prostate sampling studies show no benefit of complete embedding of RP specimens.

3. Comparison with other studies is hampered by variation in sampling protocols.

4. Complete embedding of RP specimens dramatically increases workload.

5. Biochemical recurrence is and should remain the method of choice of follow-up rather than reliance on surgical margin involvement reported by the pathology department.

Correction notice This article has been corrected since it was published Online First. The initials in the author names were missing.

Contributors EC: data collection and analysis; drafting of the manuscript and statistical analysis. EC, MK and MdB: study concept and design; acquisition of data and analysis and interpretation of data. All authors: manuscript revision.

Funding The authors have not declared a specific grant for this research from any funding agency in the public, commercial or not-for-profit sectors.

Competing interests None declared.

Patient consent for publication Not required.

Provenance and peer review Not commissioned; externally peer reviewed.

Data sharing statement Original data are available by emailing eelco@collette.nl

Open access This is an open access article distributed in accordance with the Creative Commons Attribution Non Commercial (CC BY-NC 4.0) license, which permits others to distribute, remix, adapt, build upon this work non-commercially, and license their derivative works on different terms, provided the original work is properly cited, appropriate credit is given, any changes made indicated, and the use is non-commercial. See: http://creativecommons.org/licenses/by-nc/4.0/.

\section{REFERENCES}

1. Siegel R, Naishadham D, Jemal A. Cancer statistics, 2012. CA Cancer J Clin 2012;62:10-29.

2. Mottet N, Bellmunt J, Bolla M, et al. EAU-ESTRO-SIOG Guidelines on Prostate Cancer. Part 1: screening, diagnosis, and local treatment with curative intent. Eur Urol 2017;71:618-29.

3. Meeks JJ, Eastham JA. Robotic prostatectomy: the rise of the machines or judgment day. Eur Urol 2012;61:686-7. Discussion 688689.

4. (IKNL) CCCtN. IKNL (Comprehensive Cancer Centre the Netherlands). Knowledge Institute of Medical Specialists (KiMS).
Richtlijn prostaatcarcinoom (prostate cancer guideline). 2014 https:// richtlijnendatabase.nl/richtlijn/prostaatcarcinoom/pathologisch_ onderzoek/pathologische_aspecten.html.

5. Desai A, Wu H, Sun L, et al. Complete embedding and close step-sectioning of radical prostatectomy specimens both increase detection of extra-prostatic extension, and correlate with increased disease-free survival by stage of prostate cancer patients. Prostate Cancer Prostatic Dis 2002;5:212-8.

6. Iremashvili V, Lokeshwar SD, Jorda M, et al. Prognostic implications of partial sampling of radical prostatectomy specimens: comparison of 3 methods. J Urol 2013;190:84-90.

7. Iremashvili V, Lokeshwar SD, Soloway MS, et al. Partial sampling of radical prostatectomy specimens: detection of positive margins and extraprostatic extension. Am J Surg Pathol 2013;37:219-25.

8. Sehdev AE, Pan CC, Epstein JI. Comparative analysis of sampling methods for grossing radical prostatectomy specimens performed for nonpalpable (stage T1c) prostatic adenocarcinoma. Hum Pathol 2001;32:494-9.

9. Cohen MB, Soloway MS, Murphy WM. Sampling of radical prostatectomy specimens. How much is adequate? Am J Clin Pathol 1994;101:250-2.

10. Fadul LC, da Silva MT, de Freitas LA, et al. Assessment of the circumferential margins, extraprostatic extension and Gleason score in radical prostatectomy specimens: Comparison of a partial embedding method with supplemental total inclusion of peripheral tissues. Pathol Res Pract 2016;212:217-21.

11. Grossfeld GD, Chang JJ, Broering JM, et al. Does the completeness of prostate sampling predict outcome for patients undergoing radical prostatectomy?: data from the CAPSURE database. Urology 2000;56:430-5.

12. Hall GS, Kramer CE, Epstein JI. Evaluation of radical prostatectomy specimens. A comparative analysis of sampling methods. Am J Surg Pathol 1992;16:315-24.

13. Hollenbeck BK, Bassily N, Wei JT, et al. Whole mounted radical prostatectomy specimens do not increase detection of adverse pathological features. J Urol 2000;164:1583-6.

14. Kim K, Pak PJ, Ro JY, et al. Limited sampling of radical prostatectomy specimens with excellent preservation of prognostic parameters of prostate cancer. Arch Pathol Lab Med 2009;133:1278-84.

15. Llanos CA, Blieden C, Vernon SE. Processing radical prostatectomies: an alternate-slice method is comparable with total embedding. Ann Diagn Pathol 2012;16:284-7.

16. Vainer B, Toft BG, Olsen KE, et al. Handling of radical prostatectomy specimens: total or partial embedding? Histopathology 2011;58:211-6.

17. Magi-Galluzzi C, Evans AJ, Delahunt B, et al. International Society of Urological Pathology (ISUP) Consensus Conference on Handling and Staging of Radical Prostatectomy Specimens. Working group 3: extraprostatic extension, lymphovascular invasion and locally advanced disease. Mod Pathol 2011;24:26-38.

18. Samaratunga $\mathrm{H}$, Montironi R, True L, et al. International Society of Urological Pathology (ISUP) Consensus Conference on Handling and Staging of Radical Prostatectomy Specimens. Working group 1: specimen handling. Mod Pathol 2011;24:6-15.

19. Tan $\mathrm{PH}$, Cheng L, Srigley JR, et al. International Society of Urological Pathology (ISUP) Consensus Conference on Handling and Staging of Radical Prostatectomy Specimens. Working group 5: surgical margins. Mod Pathol 2011;24:48-57.

20. Egevad L, Algaba F, Berney DM, et al. Handling and reporting of radical prostatectomy specimens in Europe: a web-based survey by the European Network of Uropathology (ENUP). Histopathology 2008;53:333-9.

21. True LD. Surgical pathology examination of the prostate gland. Practice survey by American society of clinical pathologists. Am J Clin Pathol 1994;102:572-9. 